\title{
Resonance of an imperfect joint of plates by the lowest-order symmetric Lamb mode.
}

$\operatorname{AUTHOR}(S)$ :

Mori, Naoki; Biwa, Shiro

\section{CITATION:}

Mori, Naoki ... [et al]. Resonance of an imperfect joint of plates by the lowest-order symmetric Lamb mode.. The Journal of the Acoustical Society of America 2015, 137(6): 3139-3148

ISSUE DATE:

2015-05-10

URL:

http://hdl.handle.net/2433/201592

\section{RIGHT:}

(C) 2015 Acoustical Society of America. This article may be downloaded for personal use only. Any other use requires prior permission of the author and the Acoustical Society of America. 


\title{
Resonance of an imperfect joint of plates by the lowest-order symmetric Lamb mode
}

\author{
Naoki Mori and Shiro Biwa ${ }^{\text {a) }}$ \\ Department of Aeronautics and Astronautics, Graduate School of Engineering, Kyoto University, Katsura, \\ Nishikyo-ku, Kyoto 615-8540, Japan
}

(Received 19 September 2014; revised 18 March 2015; accepted 10 May 2015)

\begin{abstract}
Resonance behavior of an imperfect joint of elastic plates subjected to the incidence of the lowestorder symmetric (S0) Lamb mode is numerically analyzed in the frequency domain by the hybrid finite element method. To this purpose, the reflection and transmission characteristics of the S0 mode are calculated for the frequency range in which the S0 mode is the only symmetric mode that can propagate in the plates. The imperfect joint is modeled as a linear spring-type interface characterized by the normal and tangential stiffnesses. As a result, it is shown that the imperfect joint of plates has two resonance frequencies at which the out-of-plane displacement amplitudes at the joint are remarkably increased. One resonance frequency depends only on the normal stiffness, and the other only on the tangential stiffness. Each resonance frequency coincides with that of a free edge of a plate, and monotonically increases with the corresponding joint stiffness. Furthermore, it is also shown that the reflection and transmission behavior of the S0 mode at the imperfect joint of the plates can be well reproduced by the one-dimensional thin-plate approximation of extensional waves when the frequency is sufficiently small compared to the resonance frequencies.
\end{abstract}

(C) 2015 Acoustical Society of America. [http://dx.doi.org/10.1121/1.4921610]

[OU]

Pages: $3139-3148$

\section{INTRODUCTION}

Elastic waves are widely utilized for the purpose of detecting and characterizing various kinds of defects in different structures that are used for transportation vehicles, civil infrastructures, power plants, etc. Lamb waves provide an effective tool for the nondestructive evaluation and structural health monitoring of plate-like structures because they can propagate relatively long distances. At the same time, however, Lamb waves have dispersive and multi-modal characteristics. This fact makes it difficult to interpret the acquired waveforms in a straightforward manner. For this reason, a number of theoretical or numerical studies have been carried out to obtain precise knowledge about the complicated behavior of Lamb waves. ${ }^{1-6}$

One of the fundamental problems of Lamb waves that have so far gained much attention is their interaction with a free edge of a semi-infinite plate that gives rise to a resonance behavior. The free-edge resonance of a plate was discovered by Shaw ${ }^{7}$ in his experimental work on titanate barium disks. Gazis and Mindlin ${ }^{8}$ numerically reproduced this phenomenon with a second-order two-dimensional plate theory for extensional waves that accounts for nonpropagating, localized modes. Torvik ${ }^{9}$ analyzed the reflection characteristics of the lowest-order symmetric (S0) Lamb mode at a free edge of a semi-infinite plate in order to explore the free-edge resonance, and showed that higherorder symmetric modes play a significant role in the resonant vibrations. Thereafter, the interaction of various Lamb modes with a free edge of a plate was analyzed by many investigators. ${ }^{10-18}$ Le Clezio et al. $^{14}$ analyzed transient

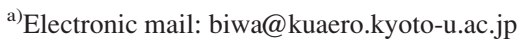

responses of the S0 mode at a free edge of a plate using the finite element method (FEM). These authors also performed experimental investigation to verify the numerical results. Pagneux ${ }^{16}$ and Zernov et al. ${ }^{17}$ obtained an approximate formula for the resonance frequency of a free edge of an isotropic plate in terms of Poisson's ratio.

Foregoing investigations have also revealed that the resonance behavior is sensitive to the boundary conditions at the plate edge. Gregory and Gladwell ${ }^{19}$ showed that a fixed edge of a plate does not show resonant vibrations like a free edge. Getman and Lisitskii ${ }^{20}$ showed that the S0-mode incidence at a specific frequency gives rise to the interfacial resonance at the joint of two elastic half-strips with different material properties. Predoi and Rousseau ${ }^{21}$ reported the resonance of an elastic plane layer between elastic strips for the S0-mode incidence.

Recently, the interaction of Lamb waves with fatigue cracks in plate structures has been studied actively in the field of structural health monitoring. ${ }^{22-24}$ When a crack is fully open, it can be modeled as a traction-free boundary as analyzed by Castaings et al., ${ }^{3}$ Gunawan and Hirose, ${ }^{6}$ etc. In realistic situations, however, fatigue cracks often have their surfaces in partial contact and constitute imperfect interfaces. The spring-type interface model is commonly employed in previous studies for describing such imperfect interfaces. In the spring-type interface model, discontinuities are allowed in the displacement components across the interface and given in terms of the corresponding stress components. This model can explain various imperfect joint conditions including a contacting interface, ${ }^{25}$ a thin bond layer between solids ${ }^{26,27}$ and a damaged interface, ${ }^{28}$ provided that the thickness of interface regions (distributed asperities, adhesive layers etc.) is sufficiently small compared to the 
wavelength. Drinkwater et $a l .{ }^{29}$ discussed the interaction of bulk waves with a partially contacting interface between solids. Pecorari ${ }^{30}$ analyzed scattering of Rayleigh waves at a surface-breaking crack with its faces in partial contact. Recently, the reflection and transmission characteristics of the lowest-order Lamb modes at an imperfect joint of plates have been analyzed by Mori et $a l^{31}$ for the low frequency range below the cut-off frequency of the first-order antisymmetric (A1) Lamb mode. It is also intriguing to investigate the possible resonance behavior of a spring-type imperfect joint of plates. To the authors' knowledge, however, this issue has not been explored yet.

The objective of the present study is to examine the resonance behavior of an imperfect joint of plates described as a spring-type interface. The paper is structured as follows. In Sec. II, the formulation is given for the time-harmonic response of an imperfect joint of isotropic elastic plates subjected to the S0-mode incidence. In Sec. III, a semianalytical approach using the hybrid finite element method $(\text { HFEM })^{31-33}$ is introduced. In Sec. IV, the resonance behavior is examined on the basis of the computed displacement amplitude at the imperfect joint, and two resonance frequencies are identified. The influence of the interfacial stiffnesses of the joint on the resonance frequencies is discussed in detail. Furthermore, the reflection and transmission behavior of the S0 mode is compared to the prediction based on a one-dimensional thin-plate approximation of extensional waves.

\section{STATEMENT OF THE PROBLEM}

As shown in Fig. 1, two semi-infinite plates $|z| \leq h$ (thickness $2 h$ ) are imperfectly jointed at $x=0$. The planestrain condition is considered in the $x-z$ plane and both plates are assumed to be made of the same linear isotropic elastic solid. The motion of the plates obeys the two-dimensional Navier equations

$$
\begin{aligned}
& (\lambda+\mu) \frac{\partial}{\partial x}\left(\frac{\partial u_{x}}{\partial x}+\frac{\partial u_{z}}{\partial z}\right)+\mu\left(\frac{\partial^{2}}{\partial x^{2}}+\frac{\partial^{2}}{\partial z^{2}}\right) u_{x}=\rho \frac{\partial^{2} u_{x}}{\partial t^{2}} \\
& (\lambda+\mu) \frac{\partial}{\partial z}\left(\frac{\partial u_{x}}{\partial x}+\frac{\partial u_{z}}{\partial z}\right)+\mu\left(\frac{\partial^{2}}{\partial x^{2}}+\frac{\partial^{2}}{\partial z^{2}}\right) u_{z}=\rho \frac{\partial^{2} u_{z}}{\partial t^{2}}
\end{aligned}
$$

where $u_{x}$ and $u_{z}$ are the displacement components, $\lambda$ and $\mu$ are Lamé's constants and $\rho$ is the mass density. The upper and lower surfaces of the plates are assumed to be tractionfree, i.e.,

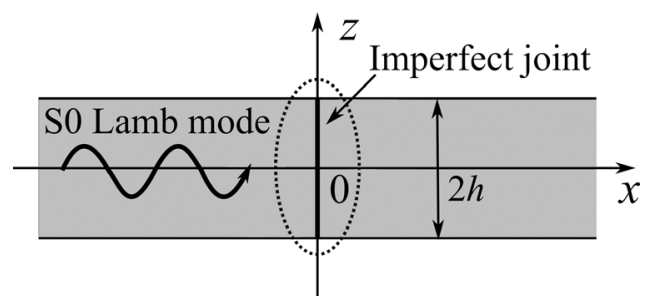

FIG. 1. Two semi-infinite isotropic plates imperfectly jointed at $x=0$.

$$
\sigma_{z z}=0, \quad \sigma_{x z}=0
$$

at $|z|=h$, where $\sigma_{z z}$ and $\sigma_{x z}$ are the stress components. A linear spring-type interface is introduced for modeling the joint of plates at $x=0$ as

$$
\sigma_{x x}^{+}=\sigma_{x x}^{-}=K_{\mathrm{N}}\left(u_{x}^{+}-u_{x}^{-}\right), \quad \sigma_{x z}^{+}=\sigma_{x z}^{-}=K_{\mathrm{T}}\left(u_{z}^{+}-u_{z}^{-}\right),
$$

where the superscripts + and - indicate the physical quantities at the right and left sides of the joint $x=0$, respectively. The parameters $K_{\mathrm{N}}$ and $K_{\mathrm{T}}$ are called normal and tangential stiffness, respectively, and represent the joint condition between a traction-free condition $\left(K_{\mathrm{N}}=K_{\mathrm{T}}=0\right)$ and a perfectly bonded condition $\left(K_{\mathrm{N}} \rightarrow \infty\right.$ and $\left.\left.K_{\mathrm{T}} \rightarrow \infty\right)\right)^{34,35}$ According to previous studies, surface conditions at the joint as well as the applied pressure have significant effect on these stiffnesses. Furthermore, existing experimental results as well as theoretical models show that the ratio of the tangential stiffness to the normal stiffness $K_{\mathrm{T}} / K_{\mathrm{N}}$ ranges from 0.2 to $0.6 .^{35-39}$ In order to elucidate the effect of interfacial stiffnesses on the resonance behavior numerically, the values of these stiffnesses are varied in a wide range in the present analysis while assuming their ratio as $K_{\mathrm{T}} / K_{\mathrm{N}}=0.2,0.4$, and 0.6 . For simplicity, both $K_{\mathrm{N}}$ and $K_{\mathrm{T}}$ are assumed to be uniform for $-h<z<h$.

The left plate is subjected to the incidence of the lowestorder symmetric (S0) Lamb mode with angular frequency $\omega$ traveling in the positive $x$ direction. The displacement fields of the incident wave are given in the complex-value representation by

$$
\begin{aligned}
& u_{x}=u_{x}^{\text {inc }} \exp (-i \omega t)=U_{+0}^{\mathrm{S}}\left(z, k_{+0}^{\mathrm{S}}\right) \exp \left(i k_{+0}^{\mathrm{S}} x\right) \exp (-i \omega t), \\
& u_{z}=u_{z}^{\text {inc }} \exp (-i \omega t)=V_{+0}^{\mathrm{S}}\left(z, k_{+0}^{\mathrm{S}}\right) \exp \left(i k_{+0}^{\mathrm{S}} x\right) \exp (-i \omega t),
\end{aligned}
$$

where $U_{+0}^{\mathrm{S}}\left(z, k_{+0}^{\mathrm{S}}\right)$ and $V_{+0}^{\mathrm{S}}\left(z, k_{+0}^{\mathrm{S}}\right)$ denote the in-plane and out-of-plane displacement profiles of the S0 mode, whose explicit forms can be found elsewhere, e.g., Refs. 6, 9, 40, and $k_{+0}^{\mathrm{S}}$ is the wave number of the S0 mode corresponding to the angular frequency $\omega$. The relation between the wave number $k$ and the angular frequency $\omega$ is given by the socalled Rayleigh-Lamb frequency equations, ${ }^{40}$ i.e.,

$$
\left(q^{2}-k^{2}\right)^{2} \tan (q h)+4 k^{2} p q \tan (p h)=0,
$$

for the symmetric modes and

$$
\left(q^{2}-k^{2}\right)^{2} \tan (p h)+4 k^{2} p q \tan (q h)=0,
$$

for the antisymmetric modes, where $p^{2}=\left(\omega / c_{\mathrm{L}}\right)^{2}-k^{2}$, $q^{2}=\left(\omega / c_{\mathrm{T}}\right)^{2}-k^{2}$, and $c_{\mathrm{L}}$ and $c_{\mathrm{T}}$ are the longitudinal and shear wave velocities, respectively. When the S0 mode interacts with the imperfect joint, partial reflection and transmission occur and the higher-order Lamb modes are generated as a result of mode conversion.

The present analysis is performed for the frequency range in which the SO mode is the only symmetric mode that can propagate in the plate. The cut-off frequency of each 
higher-order mode is commonly defined as the frequency at which the wave number is equal to zero. ${ }^{40}$ With this definition, when Poisson's ratio $\nu=\lambda /\{2(\lambda+\mu)\}$ is equal to 0.337 (3), the cut-off frequencies of the S1 and the S2 mode are $\Omega=\pi \approx 3.14$ and $\Omega=\kappa \pi / 2 \approx 3.17$, respectively, where $\Omega=\omega h / c_{\mathrm{T}}$ is the normalized frequency and $\kappa=c_{\mathrm{L}} / c_{\mathrm{T}}$ $=\sqrt{2(1-\nu) /(1-2 \nu)}$. It is noted, however, that these modes can propagate along the plate below these frequencies. Namely, according to the dispersion relation for the symmetric modes in Eq. (5), the S1 and the S2 modes can have real wave numbers and are propagating modes in the frequency range $\Omega>\Omega_{\mathrm{S} 1, \mathrm{~S} 2}=2.87$ when $\nu=0.337$ (3). Therefore, this study deals with the frequency range $\Omega<\Omega_{\mathrm{S} 1, \mathrm{~S} 2}$. For this frequency range, the $\mathrm{S} 1, \mathrm{~S} 2$, and other higher-order Lamb modes have imaginary or complex wave numbers and are localized near the joint when generated by the mode conversion.

\section{NUMERICAL ANALYSIS}

\section{A. Hybrid finite element method}

The finite element method (FEM) is a useful tool for analyzing elastic wave propagation. When applied to the problem at hand, it is suited for handling the complicated wave field that would arise near the joint, but a large number of finite elements would be necessary for covering the entire plate structure. For the purpose of efficient computation, a semi-analytical approach called hybrid finite element method $(\text { HFEM })^{31-33}$ is employed for analyzing Lamb waves interacting with an imperfect joint of plates in the frequency domain. A region surrounding the imperfect joint $(|x|<L / 2)$ is divided into finite elements, as the wave fields in $|x|<L / 2$ are expected to be complicated because of localized, non-propagating higher-order Lamb modes generated at the joint $x=0$. On the other hand, the wave fields in the outer semi-infinite regions $(|x|>L / 2)$ are represented as the modal expansions of Lamb modes. When the incident S0 mode propagates in the positive $x$ direction, the displacement fields can be expressed as

$$
\begin{aligned}
& u_{x}=u_{x}^{\mathrm{inc}}+\sum_{\alpha=\mathrm{S}, \mathrm{A}} \sum_{n=0}^{\infty} R_{\alpha n} U_{-n}^{\alpha}\left(z, k_{-n}^{\alpha}\right) \exp \left(i k_{-n}^{\alpha} x\right), \\
& u_{z}=u_{z}^{\mathrm{inc}}+\sum_{\alpha=\mathrm{S}, \mathrm{A}} \sum_{n=0}^{\infty} R_{\alpha n} V_{-n}^{\alpha}\left(z, k_{-n}^{\alpha}\right) \exp \left(i k_{-n}^{\alpha} x\right),
\end{aligned}
$$

in $x<-L / 2$, and

$$
\begin{aligned}
& u_{x}=\sum_{\alpha=\mathrm{S}, \mathrm{A}} \sum_{n=0}^{\infty} T_{\alpha n} U_{+n}^{\alpha}\left(z, k_{+n}^{\alpha}\right) \exp \left(i k_{+n}^{\alpha} x\right), \\
& u_{z}=\sum_{\alpha=\mathrm{S}, \mathrm{A}} \sum_{n=0}^{\infty} T_{\alpha n} V_{+n}^{\alpha}\left(z, k_{+n}^{\alpha}\right) \exp \left(i k_{+n}^{\alpha} x\right),
\end{aligned}
$$

in $x>L / 2$, where $u_{x}^{\text {inc }}$ and $u_{z}^{\text {inc }}$ are given in Eq. (4). The subscript $+n(-n)$ corresponds to the $n$ th-order Lamb mode traveling or decaying in the positive (negative) $x$ direction $(n=0,1, \ldots)$. The temporal factor $\exp (-i \omega t)$ is omitted in all terms on the right-hand side of Eqs. (7) and (8). The notation $\alpha=\mathrm{S}$ ( $\alpha=\mathrm{A}$ ) represents symmetric (antisymmetric) Lamb modes. The function $U_{ \pm n}^{\alpha}\left(z, k_{ \pm n}^{\alpha}\right)$ is the thickness profile of the in-plane displacement of the $n$ th-order Lamb mode, while $V_{ \pm n}^{\alpha}\left(z, k_{ \pm n}^{\alpha}\right)$ is that of the out-of-plane displacement. The wave number of the Lamb mode, $k_{ \pm n}^{\alpha}=k_{ \pm n}^{\alpha}(\omega)$, is a solution of the Rayleigh-Lamb frequency Eqs. (5) and (6). The stress components in $|x|>L / 2$ can be expressed by the modal expansions likewise. As it is impossible, however, to deal with an infinite number of Lamb modes in numerical analysis, the infinite series in Eqs. (7) and (8) are truncated at $n=N$ both for symmetric (S) and for antisymmetric (A) modes. Since the higher-order modes are localized near $x=0$, this truncation error can be kept minimal by choosing the mode number $N$ and the length $L$ appropriately.

In order to calculate the expansion coefficients $R_{\alpha n}$ and $T_{\alpha n}$ that characterize the wave fields, the continuity conditions are imposed on the displacement components $u_{x}, u_{z}$ and the stress components $\sigma_{x x}, \sigma_{x z}$ at every node at $|x|=L / 2$. As a result, a linear set of equations whose unknowns are the nodal displacements and the expansion coefficients $R_{\alpha n}, T_{\alpha n}$ $(n=0,1, \ldots, N)$ can be obtained. The singular decomposition method (SVD) is employed to determine the nodal displacements and the expansion coefficients.

\section{B. Setting of numerical parameters}

In this analysis, the plates are assumed to be made of aluminum (Lamé's constants $\lambda=56.3 \mathrm{GPa}$ and $\mu=27.1 \mathrm{GPa}$, and mass density $\rho=2700 \mathrm{~kg} / \mathrm{m}^{3}$ ) and have the thickness $2 h=1 \mathrm{~mm}$. Since numerical results are summarized in terms of normalized frequency $\Omega$, velocity ratio $\kappa$ (or Poisson's ratio $\nu$ ) and normalized interfacial stiffnesses (to be defined below), they are applicable to other plate thicknesses with appropriate scaling. Four-noded isoparametric elements whose dimensions are $2 h / 25 \times 2 h / 25$ (26 nodes in the thickness direction) are employed for discretizing the region $|x|<L / 2$.

Prior to the analysis of the imperfectly jointed plates, the reflection characteristics of the S0 mode at a free edge of the plate $\left(K_{\mathrm{N}}=K_{\mathrm{T}}=0\right)$ are analyzed to verify the accuracy of the numerical method and to determine the number of Lamb modes $N$ employed in the modal expansion in the outer regions and the inner region length $L$. Considering the energy conservation, the quantity

$$
\delta=\left|1-\frac{E_{\mathrm{R}}}{E_{\mathrm{I}}}\right|
$$

should be zero, where $E_{\mathrm{I}}$ and $E_{\mathrm{R}}$ are the energy flux of the incident wave and the reflected wave, respectively. Therefore, the value of $\delta$ defined in Eq. (9) serves as a measure of numerical errors such as the truncation error of the infinite series. ${ }^{31,33}$ After some trials, the value of $\delta$ was kept within $3.5 \%$ in the frequency range $\Omega=\omega h / c_{\mathrm{T}}<\Omega_{\mathrm{S} 1, \mathrm{~S} 2}$ by setting the numerical parameters as $N=7$ and $L=2.88 h$ (988 nodes and 900 elements).

The out-of-plane ( $z$ direction) displacement amplitude at the corner of the free edge is plotted in Fig. 2(a) as a function of the normalized frequency $\Omega$. The displacement amplitude 
is normalized with the surface out-of-plane amplitude of the incident S0 mode. In Fig. 2(a), the out-of-plane displacement amplitude takes a maximum value at $\Omega=2.37$. This frequency is identified as the resonance frequency of the free edge and is denoted by $\Omega_{\mathrm{R} 0}$. This procedure is carried out for different Poisson's ratios $\nu$. Figure 2(b) shows the variation of the free-edge resonance frequency with Poisson's ratio. According to the previous studies ${ }^{16,17}$ the normalized resonance frequency of the free edge $\Omega_{\mathrm{R} 0}$ is given by a formula

$$
\Omega_{\mathrm{R} 0}=0.652 \nu^{2}+0.898 \nu+1.9866 .
$$

The above relation for the resonance frequency is also plotted in Fig. 2(b). Comparison of the results by the HFEM with Eq. (10) shows that the relative errors are less than $0.25 \%$. This verifies the accuracy of the HFEM for calculating the resonance frequency. As a consequence, the numerical analysis for the imperfect joint of plates is carried out with the same setting of numerical parameters.

\section{RESULTS AND DISCUSSIONS}

\section{A. Resonance behavior of an imperfect joint of plates}

The interaction of the S0 mode with an imperfect joint of aluminum plates is investigated by the HFEM. For the

(a)

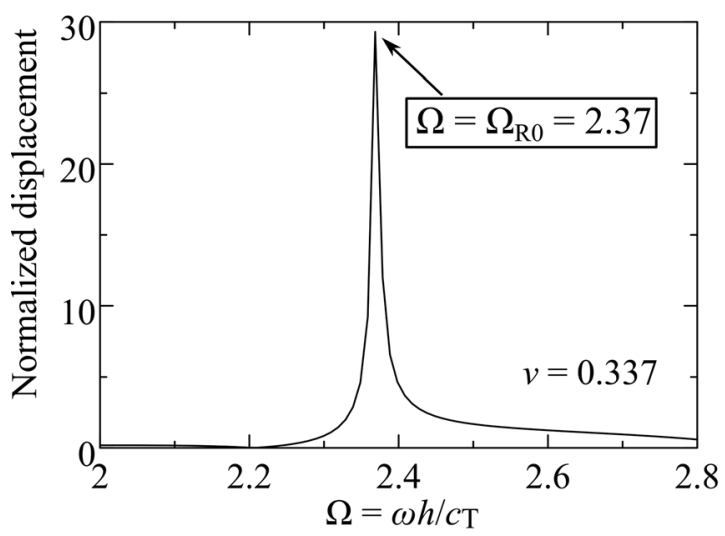

(b)

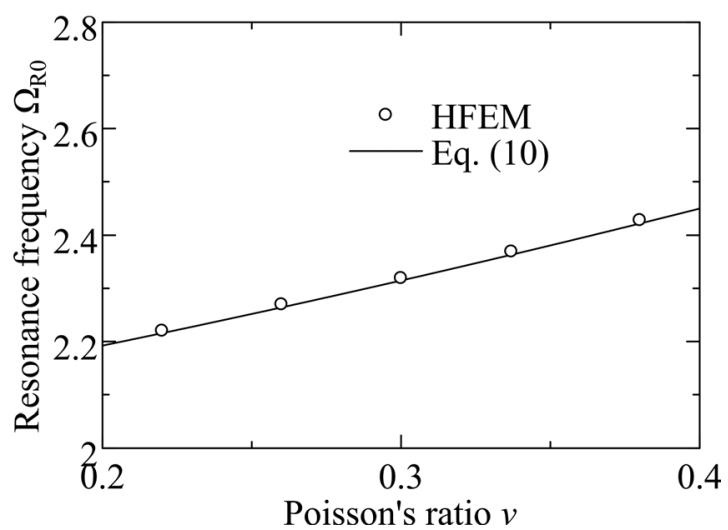

FIG. 2. (a) Variation of the normalized out-of-plane displacement amplitude at the corner of the free edge of plates $(x, z)=(0, h)$ with the normalized frequency $\Omega$. (b) Comparison of the resonance frequencies $\Omega_{\mathrm{R} 0}$ calculated in this study to Eq. (10).
S0-mode incidence, the out-of-plane displacement amplitudes at the corners of the imperfect joint $(x, z)=\left(0^{-}, h\right)$, $\left(0^{+}, h\right)$ are plotted in Fig. 3 for different normalized stiffnesses $K=K_{\mathrm{N}} h / \mu$ with the fixed ratio $K_{\mathrm{T}} / K_{\mathrm{N}}=0.2$. The displacement amplitudes are normalized by the incident amplitude. As a result, it is shown in Fig. 3(a) that the out-ofplane displacement amplitudes at the left corner of the joint $(x, z)=\left(0^{-}, h\right)$ have local maxima at two frequencies, which vary with the joint stiffnesses. Likewise, the out-of-plane displacement amplitudes at the right corner $(x, z)=\left(0^{+}, h\right)$ have two peaks at the same frequencies. In this study, the resonance frequencies of an imperfect joint of plates are obtained as the frequencies at which the out-of-plane displacement amplitude at $(x, z)=\left(0^{-}, h\right)$ has such a local peak.

The relation between the out-of-plane displacement amplitudes on the plate surface $(z=h)$ and the distance from the imperfect joint is illustrated for three normalized frequencies with the fixed stiffness $K=2$ and the ratio $K_{\mathrm{T}} / K_{\mathrm{N}}$ $=0.2$ in Fig. 4. In this case, the resonance frequencies of the imperfect joint are $\Omega=2.59$ and $\Omega=2.66$. At these frequencies, the out-of-plane displacements are remarkably increased at both sides of the joint and decay rapidly with the distance from it. As shown in Fig. 3, however, such amplification occurs only near the resonance frequencies. For example, when $K=2$ and $K_{\mathrm{T}} / K_{\mathrm{N}}=0.2$, the amplification
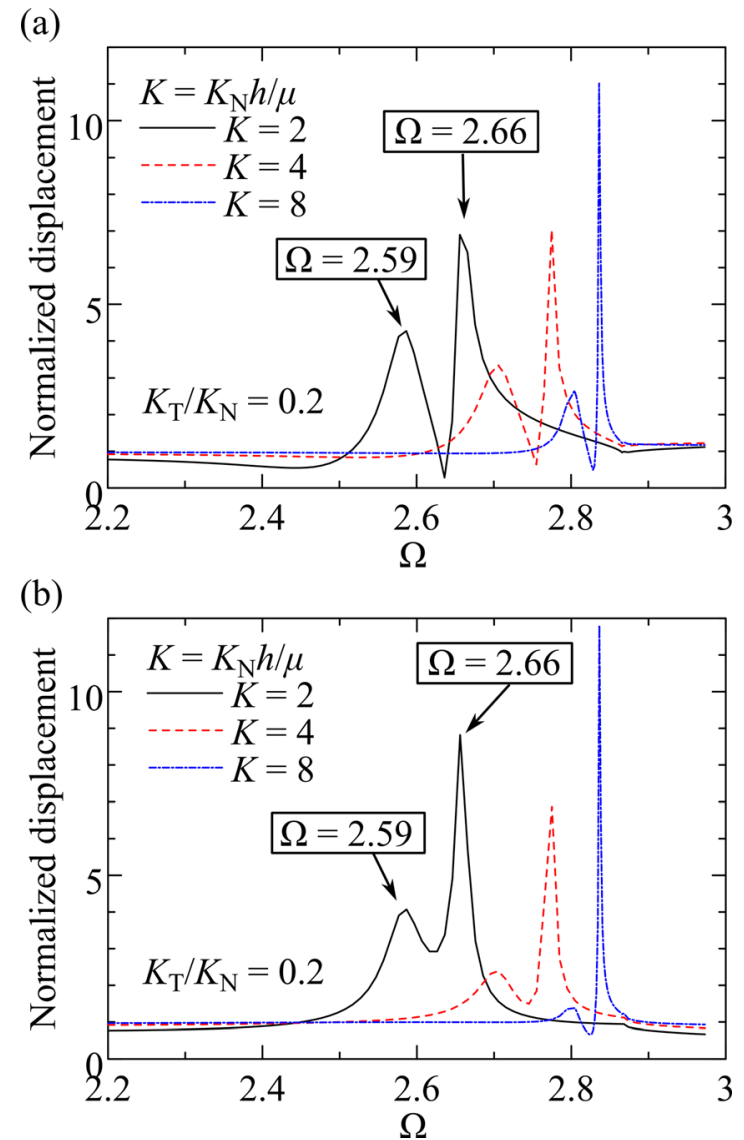

FIG. 3. (Color online) Variation of the out-of-plane displacement amplitudes at (a) the left corner $(x, z)=\left(0^{-}, h\right)$ and (b) the right corner $(x, z)=\left(0^{+}, h\right)$ of the imperfect joint of plates with the normalized frequency $\Omega$, for different normalized stiffnesses $K=K_{\mathrm{N}} h / \mu$ with the fixed ratio $K_{\mathrm{T}} / K_{\mathrm{N}}=0.2$. 
(a)

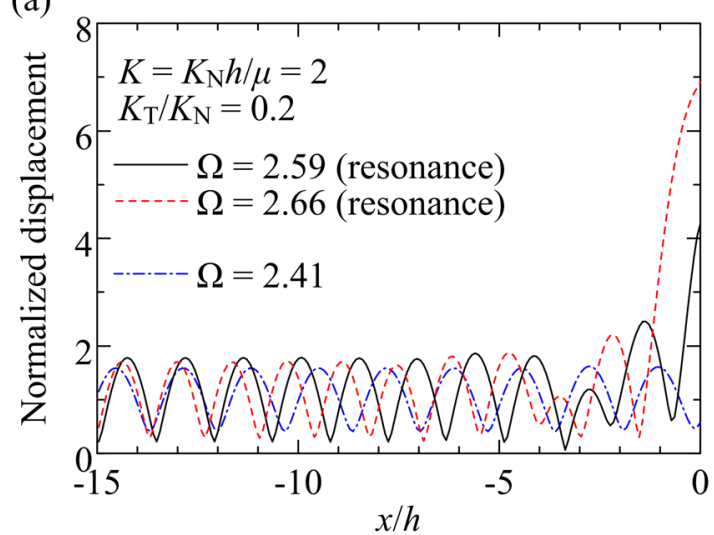

(b)

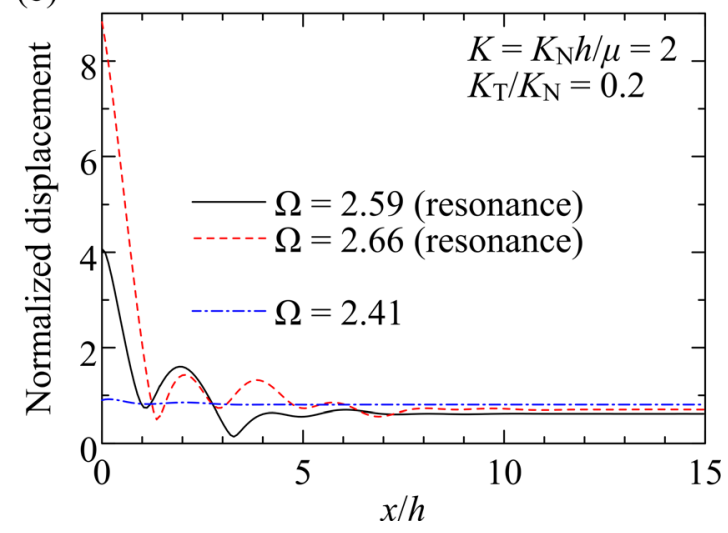

FIG. 4. (Color online) Relation of the out-of-plane displacement amplitudes on the plate surface $z=h$ with the in-plane coordinate $x / h$ in (a) $x<0$ and (b) $x>0$, for different normalized frequencies $\Omega$ when $K=2$ and $K_{\mathrm{T}} / K_{\mathrm{N}}=0.2$.

factor of the joint displacements at $x=0^{-}$is below 2 outside the frequency range $2.54<\Omega<2.74$ in Fig. 3(a), which means that the amplification effect is not significant unless the frequency is near the resonance frequencies. The amplitude distribution at $\Omega=2.41$ is shown together in Fig. 4 as an example of off-resonance behavior.

It is noted that the antisymmetric modes are not generated at the joint (i.e., $R_{\mathrm{A} n}=T_{\mathrm{A} n}=0$ for all $n$ ) because the present model is symmetric with respect to $z=0$. In addition, the S0 mode is the only symmetric mode that can propagate along the plate for $\Omega<\Omega_{\mathrm{S} 1, \mathrm{~S} 2}$. The reflection and transmission coefficients of the S0 mode are shown as functions of the normalized frequency $\Omega$ for three normalized stiffnesses with $K_{\mathrm{T}} / K_{\mathrm{N}}=0.2$ in Fig. 5. These reflection and transmission coefficients exhibit complicated behavior near the resonance frequencies of the imperfect joint.

When a free edge of a plate shows resonance behavior, the generation of localized and non-propagating higher-order symmetric modes becomes significant at the free edge. ${ }^{8-10,14,15,19}$ Analogously, it is expected that the amplitudes of higher-order symmetric modes are amplified at the resonance frequencies of an imperfect joint of plates. The relative amplitudes of the $n$ th-order symmetric modes for the S0-mode incidence are defined as

$$
\left|\frac{u_{\mathrm{S} n}^{\mathrm{R}}}{u_{\mathrm{S} 0}^{\text {inc }}}\right|=\left|\frac{R_{\mathrm{S} n} V_{-n}^{\mathrm{S}}\left(h, k_{-n}^{\mathrm{S}}\right)}{V_{+0}^{\mathrm{S}}\left(h, k_{+0}^{\mathrm{S}}\right)}\right|,
$$
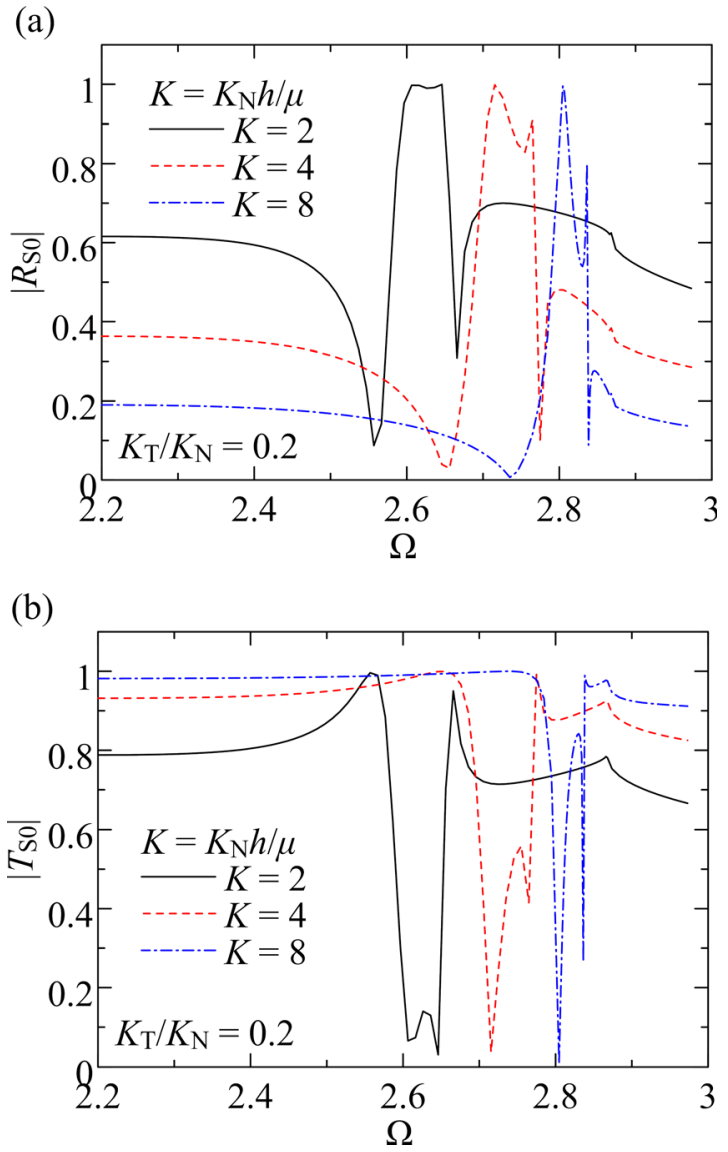

FIG. 5. (Color online) Variation of (a) the reflection coefficient $\left|R_{\mathrm{SO}}\right|$ and (b) the transmission coefficient $\left|T_{\mathrm{S} O}\right|$ of the S0 mode with the normalized frequency $\Omega$, for different normalized stiffnesses $K=K_{\mathrm{N}} h / \mu$ with the fixed ratio $K_{\mathrm{T}} / K_{\mathrm{N}}=0.2$.

for the left side, and

$$
\left|\frac{u_{\mathrm{S} n}^{\mathrm{T}}}{u_{\mathrm{S} 0}^{\mathrm{inc}}}\right|=\left|\frac{T_{\mathrm{S} n} V_{+n}^{\mathrm{S}}\left(h, k_{+n}^{\mathrm{S}}\right)}{V_{+0}^{\mathrm{S}}\left(h, k_{+0}^{\mathrm{S}}\right)}\right|,
$$

for the right side of the joint $(n=1,2, \ldots)$. The quantities defined above for the $\mathrm{S} 1(n=1)$ and the $\mathrm{S} 2$ mode $(n=2)$ are plotted in Fig. 6 when $K=2$ and $K_{\mathrm{T}} / K_{\mathrm{N}}=0.2$. As a result, the relative amplitudes of these higher-order modes increase remarkably at the resonance frequencies. Since the higherorder symmetric modes (i.e., the symmetric modes except the S0 mode) decay rapidly with the distance in this frequency range, the generation of the higher-order modes is responsible for the localized resonance of the imperfect joint.

Although the present analysis is performed in the frequency domain, in actual measurements the effect of resonance is expected to appear in the reflected or transmitted waveforms in the time-domain. In the case of the free-edge resonance, Le Clezio et al. ${ }^{14}$ have experimentally as well as numerically demonstrated that the S0 mode reflected from a free edge of a plate shows a long-time oscillation in the waveform. The reflected and transmitted S0 modes are expected to exhibit analogous behavior at the joint resonance frequencies. The corresponding experiments 
(a)

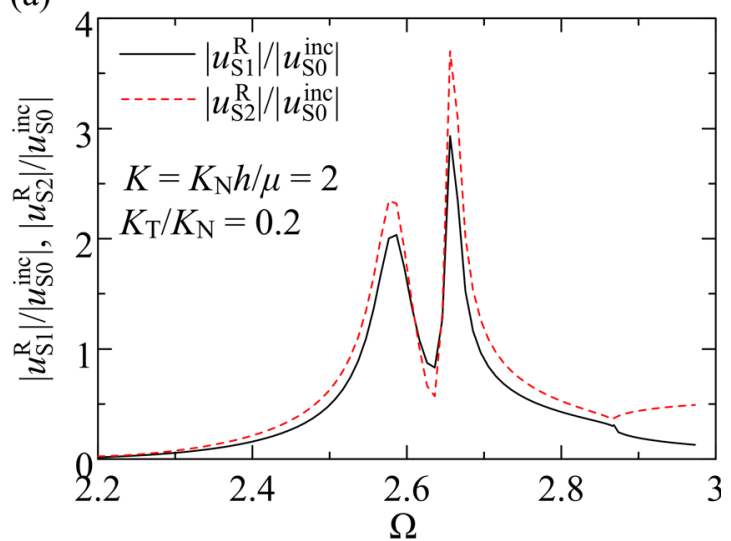

(b)

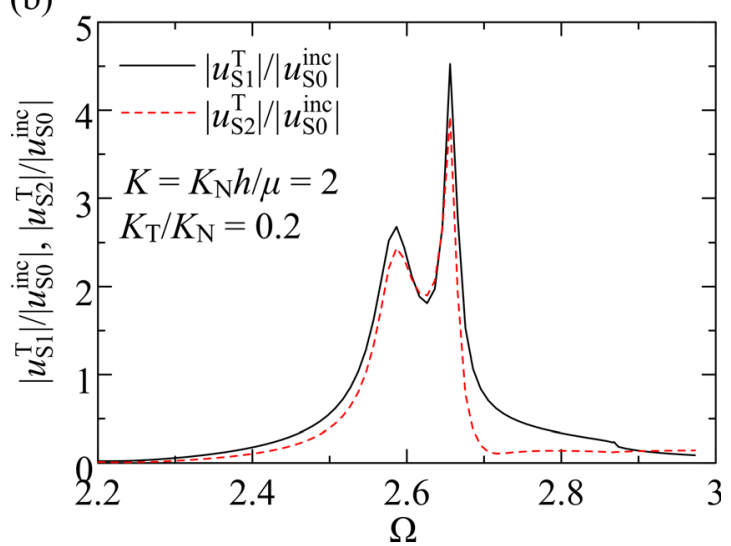

FIG. 6. (Color online) Variation of the relative amplitudes of the $\mathrm{S} 1$ and the $\mathrm{S} 2$ modes for (a) the left side and (b) the right side of the imperfect joint with the normalized frequency $\Omega$ when $K=2$ and $K_{\mathrm{T}} / K_{\mathrm{N}}=0.2$.

or numerical simulations are, however, left for future studies.

\section{B. Stiffness dependence of the resonance frequencies of an imperfect joint}

In order to single out the effect of normal stiffness $K_{\mathrm{N}}$ on the resonance characteristics of an imperfect joint of plates, the out-of-plane displacement amplitudes at the left side of the imperfect joint are plotted in Fig. 7(a) as functions of the normalized frequency $\Omega$ for different normal stiffnesses $K=K_{\mathrm{N}} h / \mu$ and fixed tangential stiffness $K^{\prime}=K_{\mathrm{T}} h / \mu=0.4$. It is shown in Fig. 7(a) that the displacement amplitudes have local maxima at two frequencies. One frequency, $\Omega=2.59$, is nominally independent of the stiffness $K$ and the other frequency varies with $K$. Hereafter, the resonance frequency that depends on $K$ is termed $\Omega_{\mathrm{RN}}$. On the other hand, the relation between the out-of-plane amplitudes of the joint and the normalized frequency $\Omega$ is shown in Fig. 7(b) for different tangential stiffnesses $K^{\prime}=K_{\mathrm{T}} h / \mu$ and fixed normal stiffness $K=2$. The displacement amplitudes have sharp peaks at two frequencies, one of which $(\Omega=2.66)$ is independent of $K^{\prime}$ while the other frequency, termed $\Omega_{\mathrm{RT}}$, varies with $K^{\prime}$.

Furthermore, the resonance frequencies of the imperfect joint are plotted in Fig. 8 as functions of (a) $K$ when $K^{\prime}=0.4$ (a)

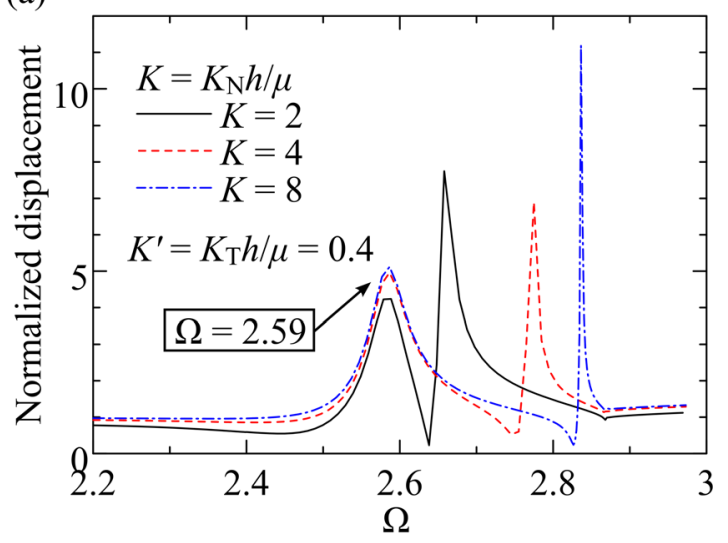

(b)

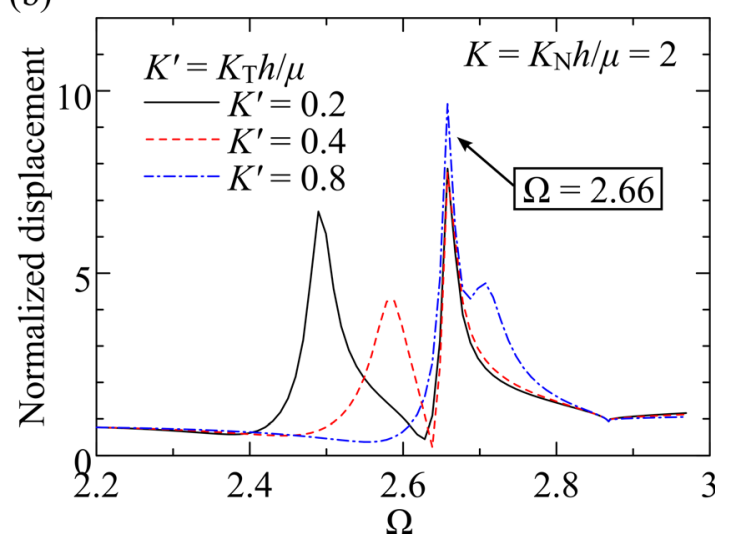

FIG. 7. (Color online) Variation of the out-of-plane displacement amplitude with the normalized frequency $\Omega$, (a) for different normalized stiffnesses $K=K_{\mathrm{N}} h / \mu$ when $K^{\prime}=K_{\mathrm{T}} h / \mu=0.4$ and (b) for different $K^{\prime}$ when $K=2$.

and (b) $K^{\prime}$ when $K=2$. In both cases, it is seen that one frequency is invariant with each joint stiffness. The other resonance frequency $\Omega_{\mathrm{RN}}\left(\Omega_{\mathrm{RT}}\right)$ increases monotonically with $K$ $\left(K^{\prime}\right)$ and eventually approaches $\Omega_{\mathrm{S} 1, \mathrm{~S} 2}$, while $\Omega_{\mathrm{RN}}$ and $\Omega_{\mathrm{RT}}$ approach the resonance frequency of the free edge, $\Omega_{\mathrm{R} 0}$, as the joint stiffnesses decrease. This selective dependence of the resonance frequencies on the joint stiffnesses can be explained, at least in a qualitative manner, by a simplistic coupled spring-mass model system as shown in the Appendix.

The relations of the resonance frequencies with the joint stiffnesses for constant stiffness ratios $K_{\mathrm{T}} / K_{\mathrm{N}}=0.2,0.4$, and 0.6 are plotted in Figs. 9(a), 9(b), and 9(c), respectively. In Fig. 9(a) where the stiffness ratio is fixed as $K_{\mathrm{T}} / K_{\mathrm{N}}=0.2$, the lower horizontal axis represents the normalized normal stiffness $K=K_{\mathrm{N}} h / \mu$ and the upper axis $K^{\prime}=0.2 K=K_{\mathrm{T}} h / \mu$. The resonance frequencies of the imperfect joint increase monotonically with the joint stiffnesses and eventually approach $\Omega_{\mathrm{S} 1, \mathrm{~S} 2}$. The resonance frequencies $\Omega_{\mathrm{RN}}$ (as a function of $K$ ) and $\Omega_{\mathrm{RT}}$ (as a function of $K^{\prime}$ ) found in Fig. 8 are plotted together in Fig. 9(a). As a result, the pair of the resonance frequencies of the joint in Fig. 9(a) coincides with the pair of $\Omega_{\mathrm{RN}}$ and $\Omega_{\mathrm{RT}}$ determined in Fig. 8. The resonance frequencies of the imperfect joint with $K_{\mathrm{T}} / K_{\mathrm{N}}=0.6$ are plotted as functions of the normalized stiffnesses in Fig. 9(c), and are also in agreement with $\Omega_{\mathrm{RN}}$ and $\Omega_{\mathrm{RT}}$. Namely, the imperfect joint has two resonance frequencies $\Omega_{\mathrm{RN}}$ and $\Omega_{\mathrm{RT}}$, one of 

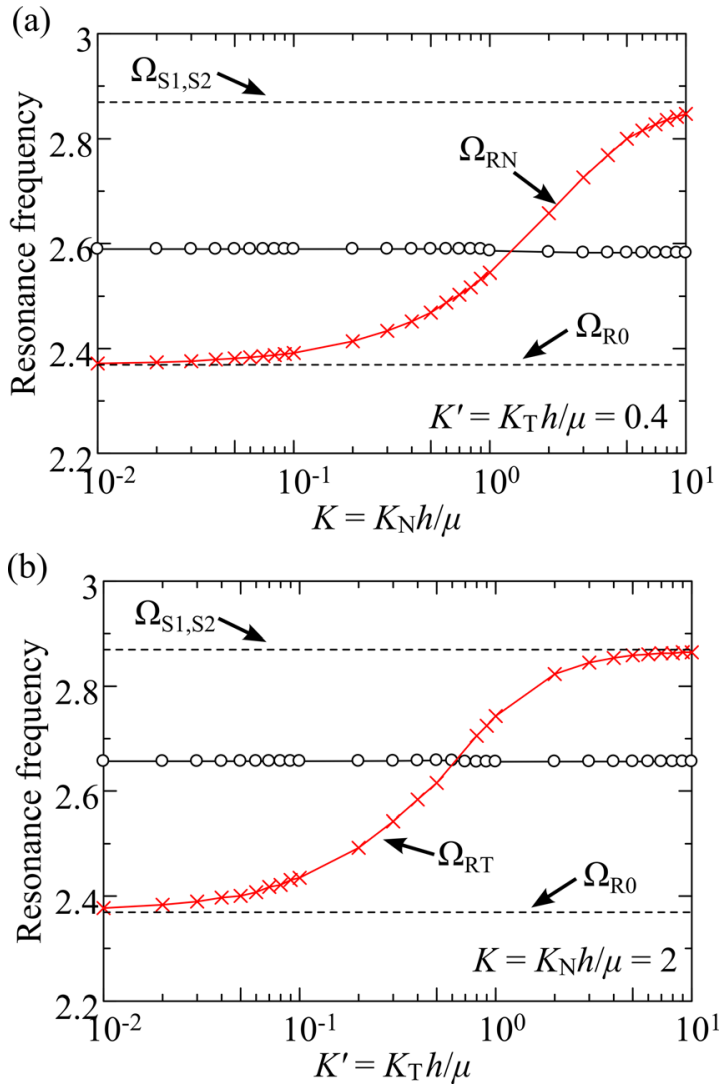

FIG. 8. (Color online) Relation of the resonance frequencies of the imperfect joint (a) with the normalized stiffness $K=K_{\mathrm{N}} h / \mu$ when $K^{\prime}=K_{\mathrm{T}} h /$ $\mu=0.4$ and (b) with $K^{\prime}$ when $K=2$.

which depends only on $K=K_{\mathrm{N}} h / \mu$ and the other only on $K^{\prime}=K_{\mathrm{T}} h / \mu$. This feature indicates the possibility to characterize the imperfect joint by measuring the resonance frequencies. It is noted, however, that the relative magnitude of $\Omega_{\mathrm{RN}}$ and $\Omega_{\mathrm{RT}}$ depends on the stiffness ratio: in the case of $K_{\mathrm{T}} / K_{\mathrm{N}}=0.6, \Omega_{\mathrm{RT}}$ is greater than $\Omega_{\mathrm{RN}}$ at each stiffness, while $\Omega_{\mathrm{RN}}$ is greater than $\Omega_{\mathrm{RT}}$ when $K_{\mathrm{T}} / K_{\mathrm{N}}=0.2$. Furthermore, the two resonance frequencies are not distinguishable for certain stiffness ratios. In Fig. 9(b) for $K_{\mathrm{T}} / K_{\mathrm{N}}=0.4$, when $K^{\prime}=0.4 K<0.24$ the resonance frequencies $\Omega_{\mathrm{RN}}$ and $\Omega_{\mathrm{RT}}$ are so close to each other and it becomes difficult to separate them.

In Fig. 10, the snapshots of the displacement fields near the joint at the resonance frequencies $\Omega=\Omega_{\mathrm{RT}}(=2.59)$ and $\Omega=\Omega_{\mathrm{RN}}(=2.66)$ are shown when $K=2$ and $K_{\mathrm{T}} / K_{\mathrm{N}}=0.2$. At $\Omega=\Omega_{\mathrm{RT}}$, the out-of-plane and the in-plane displacements at both sides of the joint vary in the opposite phase and in the same phase, respectively. In consequence, the out-ofplane displacement gap of the joint $u_{z}^{+}-u_{z}^{-}$is dominant, compared to the in-plane displacement gap $u_{x}^{+}-u_{x}^{-}$. This feature can be related to the result that the resonance frequency $\Omega_{\mathrm{RT}}$ is determined by the tangential stiffness $K_{\mathrm{T}}$. On the contrary, at $\Omega=\Omega_{\mathrm{RN}}$, the out-of-plane displacements at both sides of the joint vary in the same phase, while the inplane displacements in the opposite phase. Therefore, the response at $\Omega=\Omega_{\mathrm{RN}}$ is characterized by the in-plane displacement gap $u_{x}^{+}-u_{x}^{-}$, hence its dependence on the normal stiffness $K_{\mathrm{N}}$. (a)
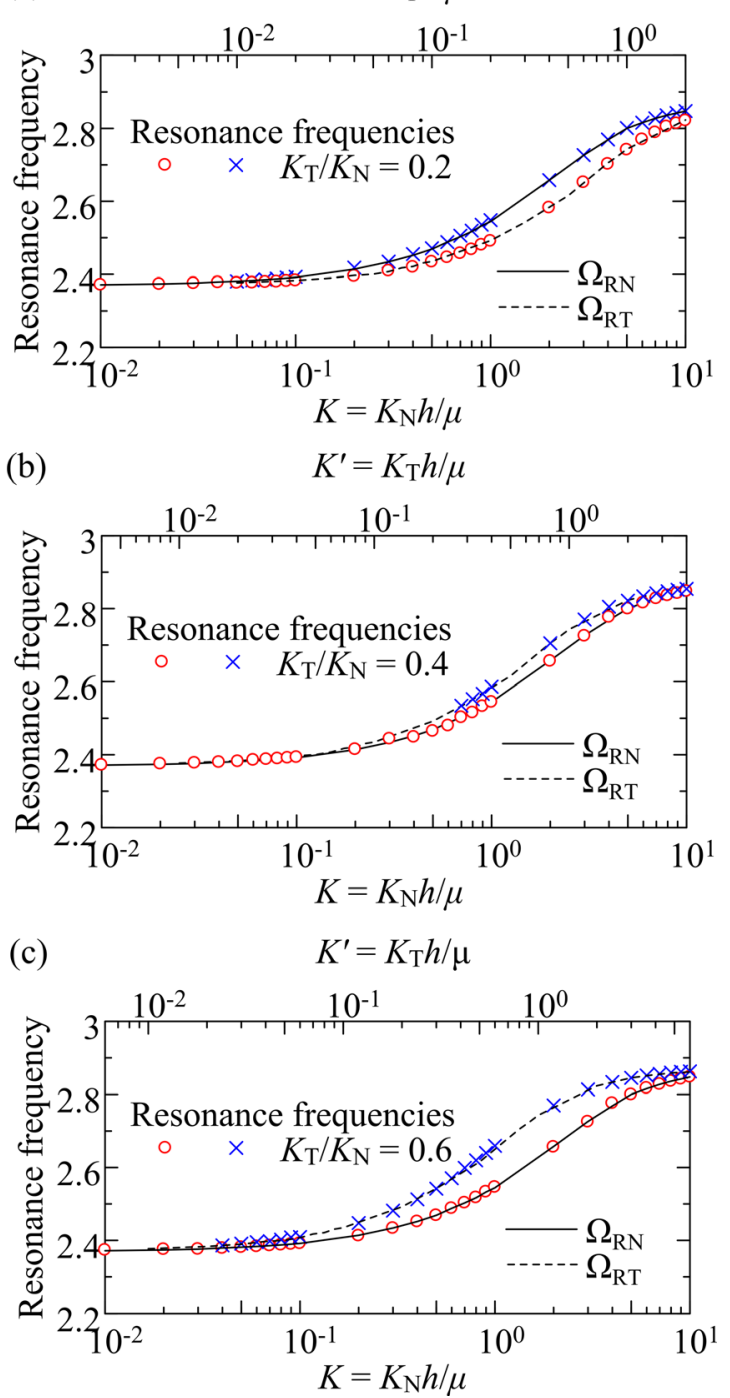

FIG. 9. (Color online) Variation of the resonance frequencies with the interfacial stiffnesses when (a) $K_{\mathrm{T}} / K_{\mathrm{N}}=0.2$, (b) $K_{\mathrm{T}} / K_{\mathrm{N}}=0.4$ and (c) $K_{\mathrm{T}} / K_{\mathrm{N}}$ $=0.6$. The upper horizontal axis represents the normalized tangential stiffness $K^{\prime}=K_{\mathrm{T}} h / \mu$, while the lower horizontal axis $K=K_{\mathrm{N}} h / \mu$. The curves for the resonance frequencies $\Omega_{\mathrm{RN}}$ and $\Omega_{\mathrm{RT}}$ found in Fig. 8 are also plotted.

\section{Reflection and transmission characteristics of the so mode}

The one-dimensional thin-plate approximation of extensional waves can explain the reflection and transmission characteristics of the S0 mode at an imperfect joint of plates in a low frequency range. ${ }^{31}$ In this approximation, the reflection and transmission coefficients of the extensional wave, $|R|$ and $|T|$, are determined by the normalized parameter $\rho c_{1} \omega / K_{\mathrm{N}}=\left(c_{1} \Omega\right) /\left(c_{\mathrm{T}} K\right)$ and do not depend on the tangential stiffness $K_{\mathrm{T}}$, where $c_{1}$ is the velocity of extensional waves in thin plates. In Fig. 11, the reflection and transmission coefficients of the S0 mode $\left|R_{\mathrm{S} 0}\right|,\left|T_{\mathrm{S} 0}\right|$ obtained by the HFEM are plotted as a function of the normalized parameter $\rho c_{1} \omega / K_{\mathrm{N}}$ with a fixed ratio $K_{\mathrm{T}} / K_{\mathrm{N}}=0.2$ for the frequency range $\Omega<\Omega_{\mathrm{S} 1, \mathrm{~S} 2}$, together with the coefficients of the approximate theory. It is shown that while excellent agreement is seen when $\rho c_{1} \omega / K_{\mathrm{N}}$ is sufficiently small, $\left|R_{\mathrm{S} 0}\right|$ and $\left|T_{\mathrm{SO}}\right|$ deviate 
(a) $\Omega=\Omega_{\mathrm{RT}}$

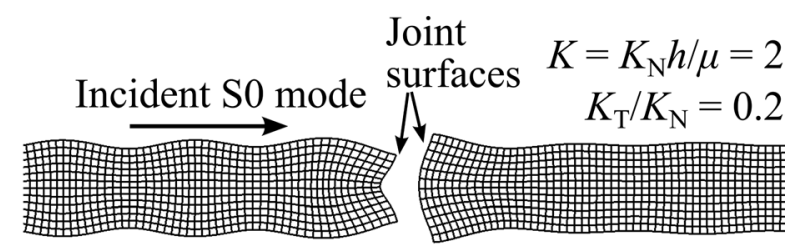

(b) $\Omega=\Omega_{\mathrm{RN}}$

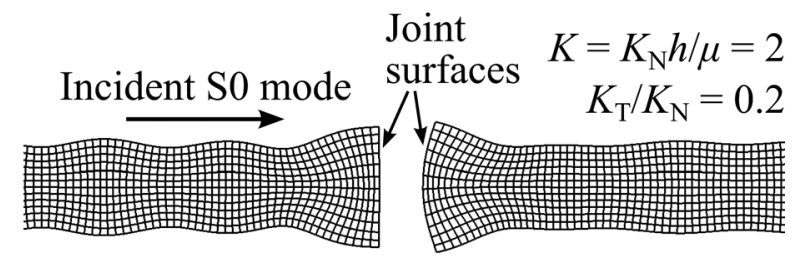

FIG. 10. Snapshots of the displacement fields of the plates near the imperfect joint at (a) $\Omega=\Omega_{\mathrm{RT}}$ and (b) $\Omega=\Omega_{\mathrm{RN}}$ when $K=K_{\mathrm{N}} h / \mu=2$ and $K_{\mathrm{T}} / K_{\mathrm{N}}$ $=0.2$, where joint surfaces are depicted separately.

from the one-dimensional model as $\rho c_{1} \omega / K_{\mathrm{N}}$ increases and approaches the resonance frequencies of the imperfect joint. Since the simple one-dimensional model does not account for the higher-order Lamb modes that are responsible for the resonance, it is not capable of describing the reflection and
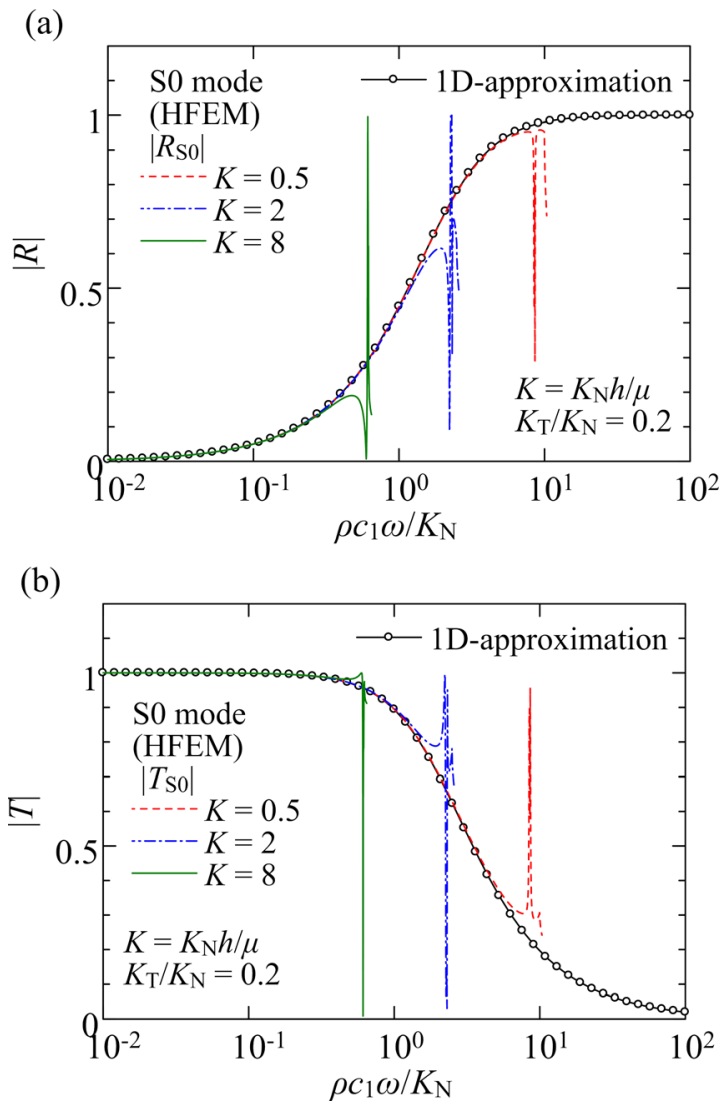

FIG. 11. (Color online) Comparison of (a) the reflection coefficient $\left|R_{\mathrm{SO}}\right|$ and (b) the transmission coefficient $\left|T_{\mathrm{S} 0}\right|$ of the S0 mode to the onedimensional approximation, for different normalized stiffnesses $K=K_{\mathrm{N}} h / \mu$ with the fixed ratio $K_{\mathrm{T}} / K_{\mathrm{N}}=0.2$. transmission characteristics of the S0 mode near and beyond the resonance frequencies.

\section{CONCLUSION}

This study has elucidated the resonance behavior of an imperfect joint of elastic plates for the incidence of the lowest-order symmetric (S0) Lamb mode. The imperfect joint has been modeled as a linear spring-type interface which is characterized by normal and tangential stiffnesses. The hybrid finite element method (HFEM) has been employed for analyzing the interaction of the incident S0 mode with the imperfect joint of plates. The analysis has been carried out for the frequency range in which the S0 mode is the only symmetric mode that can propagate along the plate. As a result, two resonance frequencies are identified at which the displacement amplitudes at the imperfect joint take local maxima. At the resonance frequencies, the amplitudes of the higher-order symmetric modes are remarkably increased and the localized resonant vibrations occur at the imperfect joint. One of the resonance frequencies depends only on the normal stiffness, while the other only on the tangential stiffness. Both resonance frequencies increase monotonically as the corresponding stiffnesses increase. Furthermore, it has been shown that one-dimensional approximation of extensional waves in a thin plate can describe the reflection and transmission behavior of the S0 mode when the frequency is sufficiently small compared to the resonance frequencies of an imperfect joint. The results of the present study show the possibility of characterizing an imperfect joint by measuring the resonance frequencies. In future studies, experimental verification is necessary for confirming the resonance behavior of an imperfect joint of plates and its applicability to the joint characterization.

\section{ACKNOWLEDGMENTS}

This work has been supported by JSPS KAKENHI Grant Numbers 26-2190 and 25289005.

\section{APPENDIX: RESONANCE OF A COUPLED SPRING-MASS SYSTEM}

The results obtained by the HFEM show that an imperfect joint of elastic plates exhibits resonance behavior for the S0-mode incidence at two frequencies, one of which is determined only by the normal stiffness and the other by the tangential stiffness. In this analysis, the edges of two plates, which have the same single resonance frequency, are coupled by linear springs in the in-plane and the out-ofplane directions. In order to qualitatively explain that the coupling of two plates leads to the double resonance frequencies having the selective stiffness dependence, a simple model of coupled spring-mass systems is introduced as shown in Fig. 12. Two bodies of the same mass $m$, connected to the rigid walls by the springs (stiffness $k$ ), are regarded to represent the resonant vibration of the plate edges. Namely, the resonance behavior of each plate edge is modeled by the spring-mass system whose natural angular frequency is $\omega_{0}=(k / m)^{1 / 2}$. The masses are connected by 


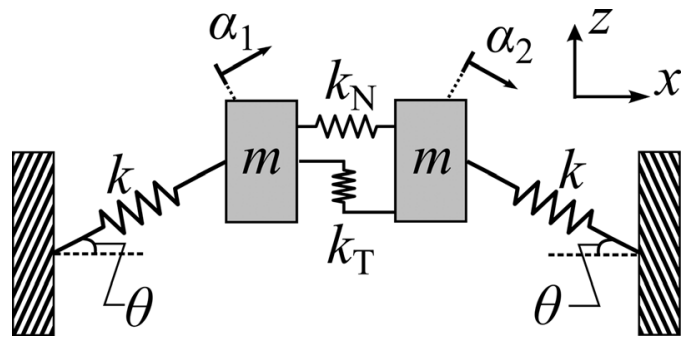

FIG. 12. Coupled spring-mass system.

two springs in the $x$-direction (stiffness $k_{\mathrm{N}}$ ) and the $z$-direction (stiffness $k_{\mathrm{T}}$ ). Each mass moves in the direction given by the angle $\theta$ with respect to the $x$ axis. The displacements of the masses in this direction, denoted by $\alpha_{1}$ and $\alpha_{2}$, obey the equations of motion

$$
\begin{aligned}
& m \ddot{\alpha}_{1}=-k \alpha_{1}-\bar{k}_{\mathrm{N}}\left(\alpha_{1}-\alpha_{2}\right)-\bar{k}_{\mathrm{T}}\left(\alpha_{1}+\alpha_{2}\right), \\
& m \ddot{\alpha}_{2}=-k \alpha_{2}-\bar{k}_{\mathrm{N}}\left(\alpha_{2}-\alpha_{1}\right)-\bar{k}_{\mathrm{T}}\left(\alpha_{1}+\alpha_{2}\right),
\end{aligned}
$$

where $\bar{k}_{\mathrm{N}}=k_{\mathrm{N}} \cos ^{2} \theta$ and $\bar{k}_{\mathrm{T}}=k_{\mathrm{T}} \sin ^{2} \theta$. By substituting a solution $\alpha_{j}=A_{j} \exp (-\mathrm{i} \omega t)(j=1,2)$ into Eq. (A1), it is shown that the vibration system has two natural angular frequencies $\omega=\omega_{\mathrm{N}}$ and $\omega_{\mathrm{T}}$, where

$$
\omega_{\mathrm{N}}=\sqrt{\omega_{0}^{2}+\frac{2 \bar{k}_{\mathrm{N}}}{m}}, \quad \omega_{\mathrm{T}}=\sqrt{\omega_{0}^{2}+\frac{2 \bar{k}_{\mathrm{T}}}{m}} .
$$

The angular frequency $\omega_{\mathrm{N}}$ depends only on $\bar{k}_{\mathrm{N}}$ (i.e., spring stiffness $k_{\mathrm{N}}$ ), while $\omega_{\mathrm{T}}$ depends only on $\bar{k}_{\mathrm{T}}$ (i.e., spring stiffness $k_{\mathrm{T}}$ ). Each natural angular frequency increases monotonically with the corresponding spring stiffnesses while approaching $\omega_{0}$ as the spring stiffness vanishes. These results qualitatively reproduce the selective dependence of the resonance frequencies on the normal and tangential stiffnesses found for the imperfect joint of plates. It is noted, however, that $\omega_{\mathrm{N}}$ and $\omega_{\mathrm{T}}$ in this simple model do not converge to finite values when the spring stiffnesses $k_{\mathrm{N}}$ and $k_{\mathrm{T}}$ diverge to infinity, while the joint resonance frequencies asymptotically approach the finite value $\Omega_{\mathrm{S} 1, \mathrm{~S} 2}$.

${ }^{1}$ D. N. Alleyne and P. Cawley, "The interaction of Lamb waves with defects," IEEE Trans. Ultrason. Ferroelectr. Freq. Control 39, 381-397 (1992).

${ }^{2}$ Y. Cho, D. D. Hongerholt, and J. L. Rose, "Lamb wave scattering analysis for reflector characterization," IEEE Trans. Ultrason. Ferroelectr. Freq. Control 44, 44-52 (1997).

${ }^{3}$ M. Castaings, E. Le Clezio, and B. Hosten, "Modal decomposition method for modeling the interaction of Lamb waves with cracks," J. Acoust. Soc. Am. 112, 2567-2582 (2002).

${ }^{4}$ T. Hayashi and K. Kawashima, "Multiple reflections of Lamb waves at a delamination," Ultrasonics 40, 193-197 (2002).

${ }^{5}$ M. J. S. Lowe and O. Diligent, "Low-frequency reflection characteristics of the $\mathrm{s}_{0}$ Lamb wave from a rectangular notch in a plate," J. Acoust. Soc. Am. 111, 64-74 (2002).

${ }^{6}$ A. Gunawan and S. Hirose, "Mode-exciting method for Lamb wavescattering analysis," J. Acoust. Soc. Am. 115, 996-1005 (2004).

${ }^{7}$ E. A. G. Shaw, "On the resonant vibrations of thick barium titanate disks," J. Acoust. Soc. Am. 28, 38-50 (1956).

${ }^{8}$ D. C. Gazis and R. D. Mindlin, "Extensional vibrations and waves in a circular disk and a semi-infinite plate," J. Appl. Mech. 27, 541-547 (1960).

${ }^{9}$ P. J. Torvik, "Reflection of wave trains in semi-infinite plates," J. Acoust. Soc. Am. 41, 346-353 (1967).
${ }^{10}$ B. A. Auld and E. M. Tsao, "A variational analysis of edge resonance in a semi-infinite plate," IEEE Trans. Sonics Ultrason. 24, 317-326 (1977).

${ }^{11}$ M. Koshiba, S. Karakida, and M. Suzuki, "Finite-element analysis of edge resonance in a semi-infinite elastic plate," Electron. Lett. 19, 256-257 (1983).

${ }^{12}$ Y. Cho and J. L. Rose, "A boundary element solution for a mode conversion study on the edge reflection," J. Acoust. Soc. Am. 99, 2097-2109 (1996).

${ }^{13}$ I. Roitberg, D. Vassiliev, and T. Weidl, "Edge resonance in an elastic semi-strip,” Q. J. Mech. Appl. Math. 51, 1-13 (1998).

${ }^{14}$ E. Le Clezio, M. V. Predoi, M. Castaings, B. Hosten, and M. Rousseau, "Numerical predictions and experiments on the free-plate edge mode," Ultrasonics 41, 25-40 (2003).

${ }^{15}$ N. Wilkie-Chancellier, H. Duflo, A. Tinel, and J. Duclos, "Numerical description of the edge mode at the beveled extremity of a plate," J. Acoust. Soc. Am. 117, 194-199 (2005).

${ }^{16} \mathrm{~V}$. Pagneux, "Revisiting the edge resonance for Lamb waves in a semiinfinite plate," J. Acoust. Soc. Am. 120, 649-656 (2006).

${ }^{17}$ V. Zernov, A. V. Pichugin, and J. Kaplunov, "Eigenvalue of a semiinfinite elastic strip," Proc. R. Soc. A 462, 1255-1270 (2006).

${ }^{18}$ M. Cès, D. Clorennec, D. Royer, and C. Prada, "Edge resonance and zero group velocity Lamb modes in a free elastic plate," J. Acoust. Soc. Am. 130, 689-694 (2011).

${ }^{19}$ R. D. Gregory and I. Gladwell, "The reflection of a symmetric RayleighLamb wave at the fixed or free edge of a plate," J. Elasticity 13, 185-206 (1983).

${ }^{20}$ I. P. Getman and O. N. Lisitskii, "Reflection and transmission of sound waves through the interfacial boundary of two joined elastic half-strips," J. Appl. Math. Mech. 52, 816-820 (1988).

${ }^{21}$ M. V. Predoi and M. Rousseau, "Lamb waves propagation in elastic plane layers with a joint strip," Ultrasonics 43, 551-559 (2005).

${ }^{22}$ J.-B. Ihn and F.-K. Chang, "Detection and monitoring of hidden fatigue crack growth using a built-in piezoelectric sensor/actuator network: I. Diagnostics," Smart Mater. Struct. 13, 609-620 (2004).

${ }^{23}$ B. C. Lee and W. J. Staszewski, "Modelling of Lamb wave interaction with open and closed fatigue cracks for damage detection," IOP Conf. Ser.: Mater. Sci. Eng. 10, 012059 (2010).

${ }^{24} \mathrm{H}$. Cho and C. J. Lissenden, "Structural health monitoring of fatigue crack growth in plate structures with ultrasonic guided waves," Struct. Health Monit. 11, 393-404 (2012).

${ }^{25}$ A. Boström and G. Wickham, "On the boundary conditions for ultrasonic transmission by partially closed cracks," J. Nondestruct. Eval. 10, 139-149 (1991).

${ }^{26} \mathrm{H}$. G. Tattersall, "The ultrasonic pulse-echo technique as applied to adhesion testing," J. Phys. D: Appl. Phys. 6, 819-832 (1973).

${ }^{27}$ A. Pilarski and J. L. Rose, "Ultrasonic oblique incidence for improved sensitivity in interface weakness determination," NDT Int. 21, 241-246 (1988).

${ }^{28}$ M. V. Golub and A. Boström, "Interface damage modeled by spring boundary conditions for in-plane elastic waves," Wave Motion 48, 105-115 (2011)

${ }^{29}$ B. W. Drinkwater, R. S. Dwyer-Joyce, and P. Cawley, "A study of the interaction between ultrasound and a partially contacting solid-solid interface,” Proc. R. Soc. London: Ser. A 452, 2613-2628 (1996).

${ }^{30} \mathrm{C}$. Pecorari, "Scattering of a Rayleigh wave by a surface-breaking crack with faces in partial contact," Wave Motion 33, 259-270 (2001).

${ }^{31}$ N. Mori, S. Biwa, and T. Hayashi, "Reflection and transmission of Lamb waves at an imperfect joint of plates," J. Appl. Phys. 113, 074901 (2013).

${ }^{32}$ M. Koshiba, S. Karakida, and M. Suzuki, "Finite-element analysis of Lamb wave scattering in an elastic plate waveguide," IEEE Trans. Sonics Ultrason. 31, 18-25 (1984).

${ }^{33}$ Y. N. Al-Nassar, S. K. Datta, and A. H. Shah, "Scattering of Lamb waves by a normal rectangular strip weldment," Ultrasonics 29, 125-132 (1991).

${ }^{34}$ J.-M. Baik and R. B. Thompson, "Ultrasonic scattering from imperfect interfaces: A quasi-static model," J. Nondestruct. Eval. 4, 177-196 (1984).

${ }^{35}$ P. B. Nagy, "Ultrasonic classification of imperfect interfaces," J. Nondestruct. Eval. 11, 127-139 (1992).

${ }^{36}$ A. Baltazar, S. I. Rokhlin, and C. Pecorari, "On the relationship between ultrasonic and micromechanical properties of contacting rough surfaces," J. Mech. Phys. Solids 50, 1397-1416 (2002). 
${ }^{37}$ D. Liaptsis, B. W. Drinkwater, and R. Thomas, "The interaction of oblique incidence ultrasound with rough, partially contacting interfaces," Nondestruct. Test. Eval. 21, 109-121 (2006).

${ }^{38}$ S. Biwa, S. Hiraiwa, and E. Matsumoto, "Stiffness evaluation of contacting surfaces by bulk and interfacial waves," Ultrasonics 47, 123-129 (2007).
${ }^{39}$ S. Biwa, S. Hiraiwa, and E. Matsumoto, "Pressure-dependent stiffnesses and nonlinear ultrasonic response of contacting surfaces," J. Solid Mech. Mat. Eng. 3, 10-21 (2009).

${ }^{40}$ J. L. Rose, Ultrasonic Waves in Solid Media (Cambridge University Press, Cambridge, UK, 1999), Chap. 8. 